\title{
La inclusión del pueblo montuvio en Ecuador a través de la democracia
}

\section{The inclusión of the montuvio people in Ecuador}

\author{
María Andrea Gonzaga \\ Universidad Pública de Navarra, Campus Arrosadía s/n, Pamplona, España. \\ E-mail: m.andreitag@hotmail.es
}

DOI: https://doi.org/10.33017/RevECIPeru2016.0015/

\section{Resumen}

En esta comunicación se describe la inclusión del pueblo montuvio en Ecuador, a través de la democracia. Se puede explicar por su complejidad étnica y el autorreconocimiento cultural como minoría que ha diversificado su mapa étnico, legitimándose en letra normativa de derechos de la etnia montuvia por parte del Estado ecuatoriano. De este modo, logra insertarse en la planificación nacional pública y, de ahí, la necesidad de un análisis crítico que permita comprender su participación histórica. En estudios recientes se establece que desde la década de los noventa se ha visibilizado el pueblo montuvio significativamente. Con la creación de varias instituciones estatales, "el primer reconocimiento que hizo el estado ecuatoriano en el 2001 de la presencia de los montuvios en el escenario político y social del Ecuador con la creación del Consejo de Desarrollo del Pueblo Montubio de la Costa Ecuatoriana y Zonas Subtropicales de la Región Litoral" [1]. Otro avance fue su reconocimiento en la Constitución del 2008, se crea el Consejo de Participación Ciudadana y Control Social y los Consejos Nacionales para la Igualdad y Participación de los Pueblos (2010) y para finalizar el reconocimiento voluntario que obtiene el ciudadano ecuatoriano al sacar su documento de identidad en el Registro civil o el ingreso de etnia de madre y padre del nacido según anuario estadístico 2013 Ecuador INEC, como un "derecho de toda persona, a decidir de manera libre y voluntaria su pertenencia a una nacionalidad o pueblo" [2], recalcando que las identidades más representativas del Ecuador son: indígena, afroecuatoriana, montuvia y otros. Lo que permite acceder a sus miembros a información separada propia de un estado plurinacional desde la perspectiva de diversidad cultural. ${ }^{1}$ Este estudio plantea determinar las etapas democráticas que atraviesa el pueblo montuvio en las últimas tres décadas, la creación de instituciones que acogen e incluyen su participación como etnia, identificando su reconocimiento atribuido por el Estado a partir de la Constitución de la República del 2008. Partiendo de la democracia deliberativa se analizan las nuevas formas de democracia participativa y comunitaria, que afloran con el reconocimiento cultural de las constituciones de la república del Ecuador de 1998 y 2008. La presente investigación se basa en una exploración bibliográfica con enfoque cualitativo que permite recopilar doctrinas y pensamientos enfocados al ámbito social y jurídico de la minoría étnica montuvia, se utilizaron varios métodos como el inductivo y deductivo, y para llegar al resultado de las interpretaciones y análisis del objeto de estudio en función a las realidades intervienen los métodos: analítico y sintético. Se pretende ofrecer algunos resultados reflejados en discursos, basados en publicaciones científicas, textos legislativos y artículos periodísticos que tributan en la redacción de conclusiones. Los resultados ponen en evidencia el progreso discursivo, el proceso histórico de la inclusión del pueblo montuvio y las etapas de democracia, fundadas en la recopilación de información que han plasmado distintos autores. Partiendo de la democracia deliberativa, las nuevas

\footnotetext{
${ }^{1}$ Es importante aclarar que el presente artículo contendrá el término montubio, en algunos casos citado textualmente, así como el Consejo de Desarrollo del Pueblo Montubio de la Costa Ecuatoriana y Zonas Subtropicales de la Región Litoral, puesto que, en la $21^{\mathrm{a}}$ edición del Diccionario de la Real Academia Española del 1992, se insertó la palabra montubio con "b" atribuyéndole la acepción: campesino de la costa, sin embargo, en la 22a edición del 2001, la 23ª edición se incluye la palabra montuvio con "v" aclarando confusiones en la escritura.
} 
formas de democracia participativa y comunitaria; y los retos del Estado como la creación de instituciones que amparan sus derechos. Como conclusiones se determina que desde la democracia deliberativa el pueblo montuvio y con la legislación del 2008 se comprende nuevos paradigmas democráticos que dinamizan la armonía y solidaridad social hacia las etnias, sin perder sus costumbres con una historia heredada, su visibilidad se ha generado con el actual gobierno de corte socialista que reconoce a través de la Constitución a los grupos étnicos, valorando su diversidad social y cultural, fortaleciendo la plurinacionalidad, de donde pueden hacer uso de sus derechos los grupos minoritarios del pueblo ecuatoriano.

Descriptores: democracia, pueblo montuvio, reconocimiento, inclusión, derechos.

\section{Abstract}

This document describes the inclusion of the montuvio people in Ecuador, through democracy. It can be explained by its ethnic complexity and cultural self-recognition as a minority that has diversified its ethnic map, legitimating itself in the law of rights of the montuvia ethnic group by the Ecuadorian State. In this way, it succeeds in inserting itself into public national planning and, the need for a critical analysis to understand its historical participation. In recent studies, it is established that since the 1990s the Montuvio people have been significantly visible. With the creation of several state institutions, "the first recognition made in 2001 by the Ecuadorian state of the presence of montuvios in the political and social scenario of Ecuador, with the creation of the Council of Development of the Montubio People of the Ecuadorian Coast and Subtropical Zones of the Coastal Region "[1]. Another sign of progress was the recognition it got by the Constitution of 2008, the creation the Council of Citizen Participation and Social Control and the National Councils for Equality and Participation of the Peoples (2010) finally, the voluntary recognition obtained by the Ecuadorian citizens when they acquire their identification card at the Civil Registration Office or the entry of ethnicity of mother and father of the new born, according to statistical yearbook 2013 Ecuador INEC, as a "right of everyone, to decide freely and voluntarily their membership of a nationality or people" [2], Emphasizing that the most representative identities of Ecuador are: indigenous, Afro-Ecuadorians, montuvia and others. This allows access to their members to information separate from a plurinational state and from the perspective of cultural diversity. This analysis proposes to determine the democratic stages that the montuvio people have undergone in the last three decades, the creation of institutions that accept and include their participation as ethnicity, identifying their recognition attributed by the State from the Constitution of the Republic of 2008. Starting from deliberative democracy that analyzes the new forms of participatory and community democracy, which arise with the cultural recognition of the constitutions of the republic of Ecuador in 1998 and 2008. This research is based, on a bibliographic exploration with a qualitative approach, that allows to collect doctrines and thoughts, focused on the social and juridical scope of the Montuvio minority ethnic. Several methods were used, such as the inductive and deductive, and to get to the result of the interpretations and analyzes of this document in function of the realities, the following methods were used: analytical and synthetic methods. It aims to offer some results reflected in speeches, based on scientific publications, legislative texts and journalistic articles that help in the writing of conclusions. The results show the discursive progress, the historical process of the inclusion of the Montuvio people and the stages of democracy, based on the collection of information that different authors have shaped. Starting from deliberative democracy, the new forms of participatory and community democracy; and the challenges of the State as the creation of institutions that protect their rights grow. As a conclusion, it is determined that from the deliberative democracy, the montuvio people and with the legislation of 2008 are understood. New democratic paradigms that dynamize the harmony and social solidarity towards the ethnic groups, without losing their customs with an inherited history, its visibility has been generated with the current government of socialist ideologies, that recognizes the ethnic groups, valuing their social and cultural diversity, strengthening the plurinationality, from where the minority groups of the Ecuadorian people can exercise their rights.

Keywords: democracy, montuvio people, recognition, inclusion, rights 


\section{Introducción}

Durante las últimas décadas, el tema de la inclusión étnica ha pasado a formar parte de la agenda de problemas sociales en Ecuador, surgiendo la necesidad de responder teóricamente a la inclusión del pueblo montuvio, a través de la democracia y las políticas públicas.

Este artículo se basa en una revisión bibliográfica y metodología cualitativa que permite confrontar ideas, pensamientos y propuestas desde diferentes perspectivas que proporcionan doctrinas para el enriquecimiento de esta exploración, enfocada al ámbito social y jurídico de una minoría étnica. Para esta investigación se utilizaron varios métodos como el inductivo y deductivo, y para llegar al análisis y a los resultados de las interpretaciones intervienen los métodos: analítico y sintético, como se refleja en la redacción de conclusiones.

Los objetivos se orientan al estudio de las etapas democráticas que atraviesa el pueblo montuvio, derechos que les corresponden, la creación de instituciones que acogen e incluyen su participación como etnia, identificando su reconocimiento atribuido por el Estado a través de las Constituciones del 98 y 2008.

Partiendo de la democracia deliberativa se analizan las nuevas formas de democracia participativa y comunitaria, que afloran con el reconocimiento cultural de las constituciones de la república del Ecuador de 1998. Además, surgen términos como plurinacional y multiétnico, mientras que en el 2008 se mantiene el término plurinacional y se reemplaza multiétnico por intercultural.

Este estudio es importante porque permite identificar los avances de un sector de la población ecuatoriana, su inserción política, inclusión, equidad social; las formas de democracia del Ecuador y avances para la etnia montuvia, como: la creación de instituciones bajo un marco jurídico como el Consejo de Desarrollo del Pueblo Montubio de la Costa Ecuatoriana y Zonas Subtropicales de la Región Litoral (CODEPMOC) (2001), el Consejo de Participación Ciudadana y Control Social y los Consejos Nacionales para la Igualdad y Participación de los Pueblos (2010).

La investigación realizada determina que en la Asamblea Constituyente del 2008 se reconoce en letras los derechos de las comunidades, pueblos y nacionalidades, resaltando distintas etnias, además de la indígena, la montuvia y afroecuatoriana.
Asímismo, refleja su derecho a participar con representantes en organismos oficiales, en la discusión de planes y políticas públicas que les afecten, para garantizar la dignidad del ser humano o de las comunidades, pueblos y nacionalidades, restituyendo sus derechos históricos. [3].

\section{Metodología.}

La investigación parte de un enfoque cualitativo por tratarse de un estudio de carácter social y de análisis bibliográfico, cuyo propósito se orientó a comprender la democracia en el pueblo montuvio del Ecuador, confrontando ideas, pensamientos y propuestas de diferentes autores como también la Constitución de la República de 1998 y 2008. Al referir la normativa se pondera el ámbito jurídico que interviene para enfatizar aspectos relevantes de la democracia.

Para obtener la información requerida se utilizaron varios métodos como: el inductivo y deductivo, aplicando un proceso sistemático y secuencial de recolección, selección, clasificación y análisis de contenidos de textos, revistas científicas, algunos textos legislativos y periodísticos; que sirvieron de fuente teórica conceptual y metodológica para llegar a conclusiones particulares y generales en función a los objetivos planteados.

\section{Fundamentación teórica}

\subsection{El nacimiento de la democracia}

La democracia supone la formación de voluntades colectivas legítimas sustentadas en el principio de soberanía popular. [4]

En Europa Occidental como cuna de la sociedad civil [5], y su intervención en américa latina, con el llamado "....al fortalecimiento de la sociedad civil a mediados de los años setenta a raíz de los golpes militares en el Cono Sur", [6], surgen nuevas estructuras sociales que acusan a un Estado de transgresor de los derechos humanos y con estos acontecimientos la sociedad empieza a buscar nuevas formas de visibilización colectiva para la democracia.

La democracia desde una perspectiva histórica es un tema muy amplio, que empezó después de la segunda guerra mundial, con luchas por la inclusión económica y política; pero sin defender aún los asuntos culturales ligados a las minorías, sobretodo de las etnias. Para explorar la idea del nacimiento de la democracia, como indica Abelardo Villegas: "América Latina es muy ilustrativo porque en pocos 
lugares como alí ha fracasado tantas veces el empeño democrático y tantas veces se ha reiterado", convirtiéndose en un ejemplo de cimentación y reestructuración de procesos democráticos [7].

En Latinoamérica, después de 30 años de transiciones políticas y reformas encaminadas a la consolidación institucional, en el siglo $X X$ se inicia el debate sobre la necesidad de profundizar la expresión democracia. En el siglo XXI la democracia deliberativa ha propiciado una discusión entre la viabilidad y deseabilidad, que intenta justificar dicha teoría deliberativa en el mundo actual.

El libro de las ilusiones de la identidad de [8], expresa el interés de una cultura democrática que no evada las diferencias, que debe existir una $<<$ política del reconocimiento $>$ ligada a los derechos humanos, sin olvidar el reconocimiento de las identidades culturales de los individuos. Para Javier de Lucas la dificultad radica en que las democracias liberales se constituyeron históricamente en EstadoNación [9], negando el pluralismo y la diversidad cultural se vivió antidemocráticamente, así mismo se legitimó la exclusión, creando un tipo de comunidad política al estilo de Procusto.

\subsection{Aparecimiento de la democracia en el Ecuador}

En el Ecuador, desde aproximadamente 1978 en la época republicana, se incorpora en los ciudadanos vocería para exigir democracia. Así como la noción democracia deliberativa fue acuñada en 1980, por Joseph M. Bessette, que la reelaboró y fundamentó de nuevo en 1994, desde aquello, cuantiosos filósofos y politólogos han contribuido a desarrollar la concepción de la democracia en el plano deliberativo. La discusión en Europa del tema de la inclusión de las minorías la abrió Jürgen Habermas, manifestando que es un término referente a un sistema político que proyecta equilibrar la democracia representativa con un proceso de toma de decisiones regido por los principios de imparcialidad y racionalidad que permitan una condición de igualdad, generando otro tipo de democracia basado en el diálogo, contrarrestando otras lógicas de democracia. Enfatiza el acto de votar como institución central de la democracia [10].

Para Sampedro-Blanco "la calidad de la democracia deliberativa dependerá de la calidad del sistema político-informativo que nutra la opinión pública" [11], dentro de sus requisitos está el acceso a la información y alfabetización, esto quiere decir que, la culpa no es solamente de un pueblo desinformado, sino también, de las instituciones que lo mantienen en dicho estado.

Otros teóricos definen la democracia deliberativa como una democracia moderna, donde las decisiones sólo pueden ser legítimas si llegan como consecuencia de una deliberación pública por parte de la ciudadanía. Se caracteriza por los esfuerzos para incluir en el mismo a grupos marginales que suelen ser ignorados en otros procesos de decisión, estos procesos pueden ser meramente representativos o meritocráticos [12].

El pensamiento de democracia deliberativa, o toma de decisiones a través de la participación de los ciudadanos, según Jaramillo. L, radica en que, "las decisiones políticas deben ser tomadas sobre la base de un proceso de deliberación pública entre los ciudadanos" [13], todo esto, mediante la incorporación de procesos colectivos en el que incluyan decisiones, basándose en el principio de deliberación que conlleva a la fundamentación y discusión pública de los planteamientos.

\subsection{La democracia étnica montuvia ecuatoriana.}

¿Pero cómo se practica esta democracia en las minorías étnicas que empezaban a visibilizarse? Entre ellas, el llamado pueblo montuvio, según censo del 2010, ya contaba con más de 1'070.728 montuvios, localizados en la región costa y litoral de forma más representativa. En este sentido, la zona montuvia se divisa claramente por sus habitantes muy bien identificados a través de su cultura montuvia que exigían derechos exclusivos [14].

Uno de los avances democráticos para la etnia montuvia fue la creación de ciertas instituciones bajo un marco jurídico como el Consejo de Desarrollo del Pueblo Montubio de la Costa Ecuatoriana y Zonas Subtropicales de la Región Litoral (CODEPMOC), que representan una respuesta contundente de la lucha de los pueblos y la irreversible reivindicación étnica mundial y nacional; bajo, Decreto ejecutivo №. 1394, en Quito, el 6 de abril del 2001, como organismo adscrito a la Presidencia de la República" [15].

EI CODEPMOC es un organismo desconcentrado y participativo, con personería jurídica, que dispone de un organismo directivo llamado Consejo Nacional, integrado de la siguiente manera: dos representantes del pueblo montuvio, por cada una de las provincias de Manabí, Los Ríos, Guayas y El 
Oro, los organismos son elegidos por asambleas provinciales del pueblo montuvio y serán calificados y registrados por el CODEPMOC; un representante del Presidente de la República; y, El Secretario Ejecutivo quien actuará con voz informativa pero sin voto. Los miembros del Consejo Nacional durarán en sus funciones cuatro años.

Asimismo, en Ecuador existe la llamada democracia participativa que se describe en varias secciones de la Constitución del 2008 con los términos "participación ciudadana". Según el Art. 207, el Consejo de Participación Ciudadana y Control Social promoverá e incentivará el ejercicio de los derechos relativos a la participación ciudadana, impulsará y establecerá mecanismos de control social en los asuntos de interés público [16].

Desde la reforma constitucional del 2008, la democracia se vio vinculada y ejemplificada en el Consejo de Participación Ciudadana y Control Social. "Tanto en la academia como en el área política se reconoce que la participación ciudadana en los procesos democráticos permite mejorar las políticas públicas" [17]. Los mecanismos de participación ciudadana tipificados en la carta magna, se refieren a: consulta popular, revocatoria de mandatos, rendición de cuentas, contraparte comunitaria para la ejecución de obras.

El Consejo de Participación Ciudadana y Control Social es un órgano integrado con representantes de los ciudadanos no designados a través de un proceso electoral, como fórmula clave de la democracia. Se prevé un singular sistema indirecto de nominación de sus integrantes, mediante un proceso de selección, al que deben someterse los postulantes que propongan las organizaciones sociales y la ciudadanía, según reza el texto constitucional.

Otra forma novedosa de democracia se configura como la democracia comunitaria, que en principio, "se relaciona con la aplicación de algunos de los derechos colectivos, es decir, el derecho colectivo de las comunas, las comunidades, los pueblos y las nacionalidades indígenas, el pueblo afroecuatoriano y los pueblos montuvio", incorporando como sujeto de derechos en el 2008, consolidándose un Estado ecuatoriano que reconoce y promueve todas las formas de organización y expresión en un marco de diversidad política y pluralismo, es donde nace el derecho a ejercer formas de consultas: consulta previa, consulta pre legislativa establecidos estos procesos en la constitución de la república [18].
Si bien es cierto que, la Constitución del 98 en la Sección primera, de los pueblos indígenas y negros o afroecuatorianos en su artículo 83, se refiere a Los pueblos indígenas, los pueblos negros 0 afroecuatorianos, como parte del Estado ecuatoriano, único e indivisible; no es hasta la constitución del 2008 que "desarrolla un concepto más amplio de derechos colectivos para el pueblo afro ecuatoriano e incorpora como sujeto de derechos, en los que sea aplicable, al pueblo montubio". Incluyéndose derechos de las comunidades, pueblos y nacionalidades en su artículo 56, las comunidades, pueblos, y nacionalidades indígenas, el pueblo afroecuatoriano, el pueblo montuvio y las comunas forman parte del Estado ecuatoriano, único e indivisible [19].

Otro organismo gubernamental que hace mérito a la democracia comunitaria desde una perspectiva étnica, son los concejos nacionales para la igualdad y participación de los pueblos, creando una condición importante para iniciar diálogos entre gobernantes y gobernados representantes de las minorías y, por ende, una dimensión propicia para la resolución de conflictos del gobierno ecuatoriano.

Como un breve antecedente se expone que el Estado ecuatoriano se ha manifestado con una tendencia occidental en busca de la homogeneización de la población a través de los tiempos, un intento de su reconocimiento cultural se da con la Constitución del 1998 en su Art. 1.- "El Ecuador es un Estado social de derecho, soberano, unitario, independiente, democrático, pluricultural y multiétnico. Su gobierno es republicano, presidencial, electivo, representativo, responsable, alternativo, participativo y de administración descentralizada" [20]. En este artículo surgen términos como plurinacional y multiétnico, como una punta de ovillo para entrar a analizar la inclusión de políticas públicas de los movimientos étnicos y su participación en la política ecuatoriana.

Mientras la constitución del 2008 plantea en su "art. 1, que Ecuador es un Estado constitucional de derechos y justicia, social, democrático, soberano, independiente, unitario, intercultural, plurinacional y laico [21]. De este modo, aparecen, los términos intercultural y plurinacional. Para el gobierno ecuatoriano la plurinacionalidad impulsa la justicia económica e igualdad, "La lucha por la igualdad es también la lucha por el reconocimiento de la diferencia" manifestando que la igualdad no equivale a homogenización, así como la diferencia tampoco equivale a desigualdad, esta lógica ha abierto nuevos paradigmas aplicables a la creación de 
jurisprudencia desde el ámbito nacional y, por ende, replicado a nivel local [22].

Respecto a cambios generados de la inclusión del pueblo montuvio, también se evidencian a nivel institucional, el registro civil a partir del 2015, incluye opciones de varias etnias, entre ellas, la montuvia, para inscribir al recién nacido y para quienes obtienen un documento nuevo de identidad.

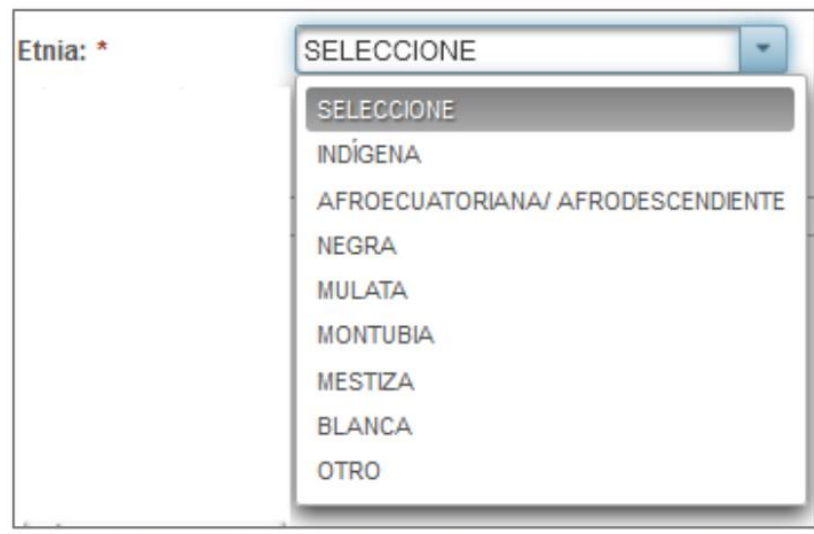

Figura 62 Opciones para registrar Etnia

Figura 1: Manual de Usuario del Sistema Nacional de Registro de Datos Vitales, [23].

Según el anuario estadístico de 2013, Ecuador INEC, se considera el "derecho de toda persona, a decidir de manera libre y voluntaria su pertenencia a una nacionalidad o pueblo", recalcando que las identidades más representativas del Ecuador son: indígena, afroecuatoriano, montuvia y otros. Lo que permite acceder a sus miembros a información separada propia de un estado plurinacional desde la perspectiva de diversidad cultural [24].

\section{Resultados y discusión}

La democracia de las etnias ecuatorianas sigue siendo frágil, por ende, se ha generado un mecanismo institucional que exige la demanda ciudadana, para contrarrestar la discriminación, siendo estratégica la voluntad estatal para los cambios legislativos que promuevan los valores democráticos de la sociedad ecuatoriana. Actualmente amparados en la Constitución de la República como una norma pionera de derechos étnicos, siendo los líderes de las etnias los principales actores de verificación y fiscalización de su cumplimiento.

Según Fuertes-Suáres, Patricia y Noboa-Chiriboga en su teoría reconocen que los montuvios componen una parte de vivencia sociopolítica y actual composición cívica y social de los costeños, que se basa en un compromiso de contribución para reconocer la rica diversidad regional, valor social, histórico, cultural y étnico de los montuvios desde el litoral [25]. Se han generado tantos cambios sociales, legislativos y políticos, ejemplo de ello existe el Plan Nacional de Desarrollo (2013-2017), instrumento al que se sujetarán las políticas, programas y proyectos públicos; la programación y ejecución del presupuesto del Estado; así como, la inversión y la asignación de los recursos públicos; además de coordinar las competencias exclusivas entre el Estado central y los gobiernos autónomos descentralizados, se demanda la creación de políticas públicas claras que contemplen una visión particular del desarrollo endógeno y asociativo del pueblo montuvio.

Por los años de 1978 y 1979 (año en que se aprobó la antepenúltima Constitución del Ecuador) se evidencia el inicio de vocerías ciudadanas, identificándose como el nacimiento de la primera etapa de democracia, acuñada por Joseph $M$. Bessette en 1980 "deliberativa", reelaborada y fundamentada en 1994, para los años de 1998 se aprobó la penúltima Constitución que marca la democracia participativa y para el 2008 (año en que se aprueba la última Constitución, la democracia deliberativa se complementa de la participativa añadida por la democracia comunitaria, fruto del actual articulado.

Hoy en día hablar de participación ciudadana o comunitaria no resulta nuevo en Ecuador, las etnias están envueltas en un ejercicio democrático deliberativo. Su apogeo ocasiona una reacción científica y periodística en los últimos años, clasificando la democracia en participativa y comunitaria.

Paralelamente a las reacciones democráticas del pueblo montuvio se crearon instituciones que amparan su derecho étnico y auto-identificación como: el Consejo de Desarrollo del Pueblo Montubio de la Costa Ecuatoriana y Zonas Subtropicales de la Región Litoral CODEPMOC (2001), el Consejo de Participación Ciudadana y Control Social y los Consejos Nacionales para la Igualdad y Participación de los Pueblos (2010).

La presión de las nacionalidades indígenas han dado pauta a las etnias ecuatorianas para exigir sus derechos; señalando que en la Constitución del 98, ya se evidenciaba la presencia de los pueblos indígenas, negros 0 afroecuatorianos, sin la presencia de los montuvios; se mantiene esta demarcación de derechos etnicos y culturales hasta 
el 2008 que el espectro etnico se extiende hacia las nacionalidades indígenas, el pueblo afroecuatoriano, el pueblo montuvio y deja abierto la opción de las comunas que forman parte del estado ecuatoriano.

No obstante, el proceso de su inclusión formal en la Constitución de la República del 2008, abre el abanico de identificación en Ecuador, marcando un hito para el pueblo montuvio y su voluntad de pertenecer a la etnia que representan; primero, con el censo del 2010 que incluye etnia montuvia, y segundo, el derecho a la auto-identificación étnica que emprende el registro civil a partir del 2015, para inscribir al recién nacido y para quienes obtienen un documento nuevo de identidad, reflejando como opción la etnia montuvia, además de la indígena, afroecuatoriana/afrodescendiente, negra, mulata, mestiza, blanca, dejando abierta la opción otras.

\section{Conclusiones}

Analizar el proceso de inserción de las etnias a través de las experiencias democráticos, permite verbalizar una democracia para el pueblo. Partiendo de la democracia deliberativa, el pueblo montuvio a la actualidad ejerce nuevas formas de democracia: participativa y comunitaria, es reconfortante que existan nuevos paradigmas democráticos en busca de armonía y solidaridad social hacia las etnias. "De modo que una nación se reconoce por sus costumbres compartidas, y por una historia heredada" [26], el Estado se ha asentado actualmente en la diversidad social, conllevado a un modelo que inserte los distintos pueblos existentes.

Se evidencia la creación de instituciones que amparan e incluyen los derechos étnicos montuvios, como: el Consejo de Desarrollo del Pueblo Montubio de la Costa Ecuatoriana y Zonas Subtropicales de la Región Litoral CODEPMOC (2001), el Consejo de Participación Ciudadana y Control Social y los Consejos Nacionales para la Igualdad y Participación de los Pueblos (2010).

Su reconocimiento atribuido por el Estado a partir de la Constitución de la República del 2008, se ratifica con el censo del 2010 que incluía la etnia montuvia, y el derecho a la auto-identificación que acciona el registro civil a partir del 2015, incluye opciones de varias etnias entre ellas la montuvia, para inscribir al recién nacido y para quienes obtienen un documento nuevo de identidad.

Y para finalizar, la construcción de un Estado radicalmente democrático fortalecido en la plurinacionalidad, es solo un paso inicial del inicio de la construcción de una sociedad que vela por métodos más transparentes en el desarrollo de los Gobiernos de turno. El rol principal hegemónico en la participación deliberativa lo juegan las organizaciones sociales, especialmente aquellas enraizadas en lo local, étnico y cultural; pues las distintas formas de empoderamiento y proceso de toma de decisiones territoriales deben respetarse en base a las discusiones de la sociedad civil en general, como el conjunto de ciudadanas y ciudadanos miembros de un país, unitario, intercultural, plurinacional.

\section{Agradecimientos}

Se agradece a Luis Alvarado persona representativa del pueblo montuvio, político que integró comunidades de diferentes provincias del Ecuador que contribuyen a la organización nacional montuvia, por ser la persona y activista que ha motivado significativamente mi interés por la historia montuvia; a miembros del Codepmoc que han aportado con sus conocimientos y experiencias de participación democrática.

\section{Referencias}

[1] L. Ribadeneira, Los Montubios: Sujetos étnicos en construcción. Facultad Latinoamericana de Ciencias Sociales (2013), p. 87.

[2] INEC. Instituto Nacional de Estadísticas y Censos. Anuario estadístico, Quito, (2013), p. 30.

[3] Ecuador. Asamblea Constituyente. (2008a). Constitución de la República del Ecuador. Recuperado a partir de http://www.asambleanacional.gov.ec/docum entos/constitucion de bolsillo.pdf (2008a) p. 31.

[4] J. Habermas, La soberania popular como procedimiento. En facticidad y validez. Trotta, Madrid, (1999), p 239.

[5] S. Giner, La sociedad civil en Europa Occidental, (1987), 1. 49-56. p.1.

[6] N. Lechner, La (problemática) invocación de la sociedad civil. Perfiles latinoamericanos, (1994), 131-144. p.132.

[7] M. Rivara De Tuesta, Filosofía e historia de las ideas en el Perú. (2000). p.93

[8] P. Gómez García, Las ilusiones de la identidad, Madrid, 2000; José A. Pérez Tapias, “¿Podemos juzgar las culturas?”, en J.B. Llinares-N. Sánchez Durá (eds.), Filosofía de la cultura, Valencia, (2001), 551-565. p.81. 
[9] J. de Lucas, Prejuicios en el uso político de las identidades culturales. (2003). p.54.

[10] J. Habermas, "El Estado democrático de derecho. Una unión paradójica de principios contradictorios, en anuario de derechos humanos (Madrid, Instituto de derechos Humanos, Universidad Complutense), (2001), pp.435-458.

[11] V. Sampedro-Blanco, Opinión pública y democracia deliberativa: medios, sondeos y urnas. International Journal of media and Cultural Politics, (2000) 3.1. p.28.

[12] J.Elster, La democracia deliberativa, Barcelona, Gedisa, (2001).

[13] L. Jaramillo, La democracia deliberativa a debate. Cuadernos de Investigación, (2012), (85). p..21.

[14] INEC, "Las Cifras de los Pueblos Afroecuatoriano, Montubio e Indígena. Una mirada desde el Censo de Población y Vivienda 2010". Quito: Instituto Nacional de Estadística y Censo (INEC)-Comisión Nacional de Estadísticas para Pueblos Indígena, Afroecuatorianos y Montubios (CONEPIA)- CODENPE-CODAECODEPMOC,http://www.ecuadorencifras.go b.ec//resultados/, (2011). p.30.

[15] Ecuador.Gobierno. Decreto Presidencial N ${ }^{\circ}$ 1394 (2001).

[16] Ecuador. Asamblea Constituyente. (2008b). Constitución de la República del Ecuador. Recuperado a partir de http://www.asambleanacional.gov.ec/docum entos/constitucion de bolsillo.pdf, (2008b). p. 109.

[17] S. García, Buenas Prácticas en Participación, (2008).
[18] P. Gonzalez, La democracia representativa, directa y comunitaria, La hora, (2012).

[19] T. Arias, Ecuador un estado constitucional de derechos, Instituto de investigación y debate sobre la gobernanza, (2008).

[20] Ecuador. Congreso Nacional. Constitución política de la república de Ecuador, (1998). p.1.

[21] Ecuador. Asamblea Constituyente, Constitución de la República del Ecuador. Recuperado a partir de http://www.asambleanacional.gov.ec/docum entos/constitucion de bolsillo.pdf, (2008c). p.15.

[22] R. Ramírez, Los nuevos retos de América Latina: Socialismo y Sumak Kawsay. SENPLADES. Quito, (2010). p. 25.

[23] Registro Civil, Manual de Usuario del Sistema Nacional de Registro de Datos Vitales, Quito, (2015). p. 17.

[24] INEC, Instituto Nacional de Estadísticas y Censos. Anuario estadístico, Quito, (2013).

[25] P. Fuertes-Suáres, \& E. Noboa-Chiriboga, La cultura montubia en el proceso de enseñanza - aprendizaje. Milagro. (2011). p.80.

[26] J. A. Sánchez, Revista de Antropología Experimental El modelo de Estado plurinacional en Ecuador: Ideas y reflexiones, 91-107, (2014). p. 92. 